\title{
ECHINACEA/SAge OR CHLORHEXIDINE/Lidocaine FOR Treating Acute Sore Throats: A Randomized Double-Blind Trial
}

\author{
A. Schapowal ${ }^{1}$, D. Berger ${ }^{2 *}$, P. Klein ${ }^{3 *}$, A. Suter ${ }^{4 *}$ \\ ${ }^{1}$ Allergy Clinic, Landquart, Switzerland; ${ }^{2}$ Schüpfen, Switzerland; ${ }^{3}$ d.s.h. statistical services GmbH, Rohrbach, Germany; \\ ${ }^{4}$ Medical Department, A. Vogel Bioforce AG, Roggwil, Switzerland
}

\begin{abstract}
Background: The aim of this trial was to assess the relative efficacy of a sage/echinacea spray and a chlorhexidine/lidocaine spray in the treatment of acute sore throats.

Methods: This was a multicenter, randomized, doubleblind, double-dummy controlled trial carried out in eleven general practices in Switzerland. A total of 154 patients (133 analyzed in per protocol collective) at least 12 years old with acute sore throat present for not more than 72 hours prior to inclusion and with a throat score $\geq 6$ participated in the study. They used either an echinacea/sage spray or a chlorhexidine/ lidocaine spray with two puffs every 2 hours, in a doubledummy blinded manner, up to 10 times daily until they were symptom-free, for a maximum of 5 days. The main outcome measures was the comparison of response rates during the first three days. A response was defined as a decrease of at least $50 \%$ of the total symptoms compared to baseline.

Results: The echinacea/sage treatment exhibited similar efficacy to the chlorhexidine/lidocaine treatment in reducing sore throat symptoms during the first 3 days $(\mathrm{P}(\mathrm{x}<\mathrm{Y})=.5083)$. Response rates after 3 days were $63.8 \%$ in the echinacea/sage group and $57.8 \%$ in the chlorhexidine/lidocaine group. For all secondary parameters, such as time to becoming symptom free, throat pain, and global assessments of efficacy by the physician and patient, no difference between the two treatments was seen. They were both very well tolerated.

Conclusion: An echinacea/sage preparation is as efficacious and well tolerated as a chlorhexidine/lidocaine spray in the treatment of acute sore throats.
\end{abstract}

Key words: Clinical trial - sore throat - echinacea salvia - chlorhexidine - lidocaine

\section{INTRODUCTION}

Acute sore throats are one of the most commonly seen conditions in general practice, rating as the third most common disease to be treated in the United States [1]. The disease encompasses any upper respiratory tract infection most often caused by viruses or

\footnotetext{
* These authors contributed equally to this work.
}

bacteria in which pain in the throat is the predominant symptom [2], mostly accompanied by inflammation and swelling of the pharyngeal region. The total costs for treatment and missed work are estimated in the United States to be $\$ 1.3$ billion annually. Only about $10-20 \%$ of patients with a sore throat are streptococcus positive in the throat. Most patients have viral infections and benefit from symptomatic treatment alone [3]. Pain relief is one of the main reasons a patient with a sore throat sees a doctor, and NSAIDs such as paracetamol, ibuprofen [4], diclofenac, and acetylsalicylic acid [5] are taken, or anesthetic substances such as lidocaine are used. Additionally, substances such as chlorhexidine, with a broad antimicrobial activity, are in use [6]. Further treatment options are herbal medicinal products that patients often use to ease symptoms.

The purple coneflower, Echinacea purpurea, is a medicinal plant with a long tradition of being used as a remedy in the treatment of upper respiratory tract infections, and a recent review confirmed the efficacy of echinacea preparations in the treatment and prophylaxis of these infections [7]. The plant has anti-inflammatory and immune modulatory properties that seem to be mediated by endocannabinoid receptor CB2-induced upregulation of TNF- $\alpha$ mRNA[8]; in addition, antiviral and antibacterial properties have been reported [9].

Another medicinal plant that has been used traditionally and is also recommended for use in infections and inflammations of the mouth is the common sage, Salvia officinalis. Sage compounds have anti-inflammatory, antibacterial, antinociceptive, and astringent properties [10]. Because both plants are traditionally used in the treatment of upper respiratory tract infections and for sore throats, we wanted to see if an herbal treatment could exhibit an efficacy similar to that of synthetic compounds. In this trial, we compared the efficacy of an echinacea/sage throat spray to that of a chlorhexidine/lidocaine spray in the treatment of acute sore throats.

\section{Participants, Methods and Statistics \\ PARTICIPANTS}

Patients were recruited among 11 general practices in Switzerland from February to August 2006. The study was approved by the relevant ethical committees and 
the Swiss health authority Swissmedic and was carried out according to the guidelines of Good Clinical Practice and the ethical obligations of the Declaration of Helsinki.

Inclusion criteria were age $>12$ years; acute pharyngitis or tonsillitis with symptoms of throat pain and inflammation of the pharynx and/or tonsils; onset of sore throat less than 72 hours before inclusion into the study; a Tonsillopharyngitis Severity Score $\geq 6$; and written informed consent.

Exclusion criteria included the use of the following within the respective time frames preceding the start of the study: analgesics $<12$ hours; antibiotics $<24$ hours; topical throat pain medication $<4$ hours; or systemic corticosteroids within the last month. Other exclusion criteria were symptoms of primary bacterial pharyngitis or secondary bacterial infection; serious illness such as tumors; allergy to one of the ingredients; pregnancy or lactation; hypersensitivity to ibuprofen; or participation in another clinical trial in the previous 30 days.

\section{TREATMENT AND BLINDING}

Patients received either a 50-ml bottle of an echinacea/sage spray containing an aqueous alcoholic $(57.3 \% \mathrm{~m} / \mathrm{m}$ ethanol) fresh-plant extract of E. purpurea $(95 \%$ aerial parts and $5 \%$ root) and of leaves of S. officinalis $(863.3 \mathrm{mg} / \mathrm{ml}$ E. purpurea flowering aerial parts tincture, Drug Extract Ratio 1:12; $45.5 \mathrm{mg} / \mathrm{ml}$ E. purpurea root tincture DER 1:11; and 430.0 $\mathrm{mg} / \mathrm{ml} \mathrm{S}$. officinalis leaves tincture DER 1:17; batch no. 019182) or a spray containing $1 \%$ chlorhexidine gluconate and $2 \%$ lidocaine hydrochloride (Collunosol $^{\circledR}$, Sanofi AG). The echinacea tinctures (Echinaforce ${ }^{\circledR}$ ) were used as a concentrate, but the sage tincture was used without previous evaporation to maintain the valuable sage essential oils.

Placebos were produced with inert flavoring substances from Günther Aromen GmbH, Beinwil a. S., Switzerland. Because this was a double-dummy blinded study, each patient also received with the verum a placebo corresponding to the other spray treatment that was similar in appearance, taste, and smell. The verum and the placebo bottles were issued in a sealed box, together with a package of 20 tablets of ibuprofen $200 \mathrm{mg}$ that served as rescue medication if pain symptoms were too severe.

To check blinding at the end of the treatment, patients were asked to guess which bottle contained the treatment.

Compliance was checked with weighing of the returned bottles and the count of used rescue medication, and patients documented in a diary how many times they had applied each spray daily.

Patients had to apply spray every 2 hours with two puffs to the pharyngeal area up to a maximum of 10 times daily. Treatment duration was until illness was resolved or for a maximum of 5 consecutive days.

\section{PRIMARY PARAMETER}

Patients filled out a diary three times daily (morning, midday, evening), recording the Tonsillopharyngitis
Severity Score [11], which consists of the symptoms of throat pain, difficulty in swallowing, salivation, erythema, and fever (rated on a 4 -point scale: $0=$ none, 1 $=$ mild, $2=$ moderate, $3=$ severe $)$.

The primary parameter was defined as the comparison of the response rates of the two treatment groups after the first, second, and third days. A response to treatment is defined as a reduction by $50 \%$ of the total baseline score taken prior to treatment start.

\section{SECONDARY PARAMETER}

The secondary parameters for assessing efficacy were a comparison of response rates after the fourth and fifth days of treatment; reduction of the total symptom score (throat pain, difficulty swallowing, salivation, erythema, and fever symptoms) after days 1 to 5 compared to the baseline score; time taken to become symptom free (total score $\leq 2$ ) within the 5-day treatment period; percentage of patients with a resolution of illness by day 5 (objective assessment by physician, total score $\leq 2)$; Visual Analogue Scale VAS for throat pain; number of ibuprofen tablets used; and global assessment of efficacy by the physician and patient.

Safety parameters considered were frequency of adverse events, global assessment of tolerability by physician and patient, vital parameters (pulse, blood pressure, body temperature), and laboratory blood values [ALAT (GPT, ALT), ASAT (GOT, ALT), total bilirubin, plasma glucose, serum creatinine, ESR (1 h), CRP, total cholesterol, erythrocytes, MCHC, MCH, MCV, hematocrit, hemoglobin, leukocytes, and thrombocytes].

\section{Sample Size, Randomization, And Statistical ANALYSIS}

The Mann-Whitney statistic $\mathrm{P}(\mathrm{x}<\mathrm{Y})$ was used as a measure of relevance of group differences as being the associated effect size measure for the WilcoxonMann-Whitney test. It is defined as the probability that a randomly selected patient from the test group is better off than a randomly selected patient from the reference group. The maximum acceptable difference (non-inferiority region) was predefined by means of Mann-Whitney statistics as $\mathrm{P}(\mathrm{X}<\mathrm{Y})=0.36$ (mediumsized/relevant difference).

With a non-inferiority margin of $\mathrm{P}(\mathrm{X}<\mathrm{Y})=0.36$ and a one-sided test of non-inferiority at a level of significance of $\alpha=0.025$ and a power of $80 \%, 67$ evaluable patients in each treatment group were needed. With respect to dropouts and because the per protocol collective was the population analyzed for the primary parameter, a total of 150 patients had to be recruited.

Randomization codes were computer-generated in blocks of four (RanCode, Version 3.6, IDV-Gauting, Germany) with a randomization ratio of $1: 1$.

The null hypothesis stated that chlorhexidine/lidocaine reaches at least a medium-sized superiority to the sage/echinacea spray. The alternative hypothesis stated that sage/echinacea is on at least one of the first three days less than medium-sized inferior to chlorhexi- 
dine/lidocaine. The test of the three efficacy criteria for non-inferiority was performed using a generalized Wilcoxon-Mann-Whitney test (directional test) according to Wei-Lachin[12] with $\alpha=0.025$, one-sided. If the combined global test gave a significant result then efficacy was proven in a confirmatory way (at least one null hypothesis of a null difference cannot be true). If the summarizing test gave a significant result, then the three single efficacy criteria could be tested univariately in a top-down procedure with full alpha according to the closed testing principle. The confirmatory tests for non-inferiority were performed applying the "confidence interval approach."

The non-inferiority of echinacea/sage compared to chlorhexidine/lidocaine could be accepted, if the lower bound of the one-sided $97.5 \%$ confidence interval of the Mann-Whitney statistic was greater than 0.36 , corresponding to an overall two-sided level of significance of $\alpha=0.05$. Confidence limits were calculated using the validated test system TESTIMATE (Version 6.4).

Secondary parameters were analyzed in a descriptive manner with $95 \%$ confidence intervals.

\section{RESUlts}

\section{Patient Characteristics and Flow through the STUDY}

A total of 154 patients were screened, randomized, and treated, and they formed the safety population. There were 80 patients allocated to the echinacea/sage group and 74 to the chlorhexidine/lidocaine group.
Two patients in the echinacea/sage and one in chlorhexidine/lidocaine were lost to follow-up, and in each group nine patients were excluded from the per protocol population mainly because of incorrect use of the study medication; this population finally consisted of 133 patients (Fig. 1).

The baseline characteristics between treatment groups were comparable, with only a significant difference found for age $(\mathrm{P}<0.05)$, with the chlorhexidine/lidocaine group having younger patients (Table 1). The throat score was somewhat higher in the chlorhexidine/lidocaine group. The average study duration was 5.6 days in the echinacea/ sage and 6.4 days in the chlorhexidine/lidocaine group.

\section{Compliance AND Blinding}

The patients in the per protocol population in the echinacea/sage group used an average $11.7 \pm 6.4 \mathrm{~g}$ of the verum spray and $7.8 \pm 5.4 \mathrm{~g}$ of the placebo spray; the chlorhexidine/lidocaine group used $10.1 \pm 6.9 \mathrm{~g}$ of the verum and $14.2 \pm 10.5 \mathrm{~g}$ of the placebo spray. Even though more of the spray was consumed in the chlorhexidine/lidocaine group, this difference was not statistically significant $(\mathrm{P}=.109)$.

The blinding question showed that in the echinacea/sage group, $78.3 \%$ of the patients guessed the verum correctly and $75.4 \%$ of the patients guessed the placebo correctly. In the chlorhexidine/lidocaine group, only $26.6 \%$ guessed the verum correctly and $70.3 \%$ thought their echinacea/sage placebo was actually the verum.

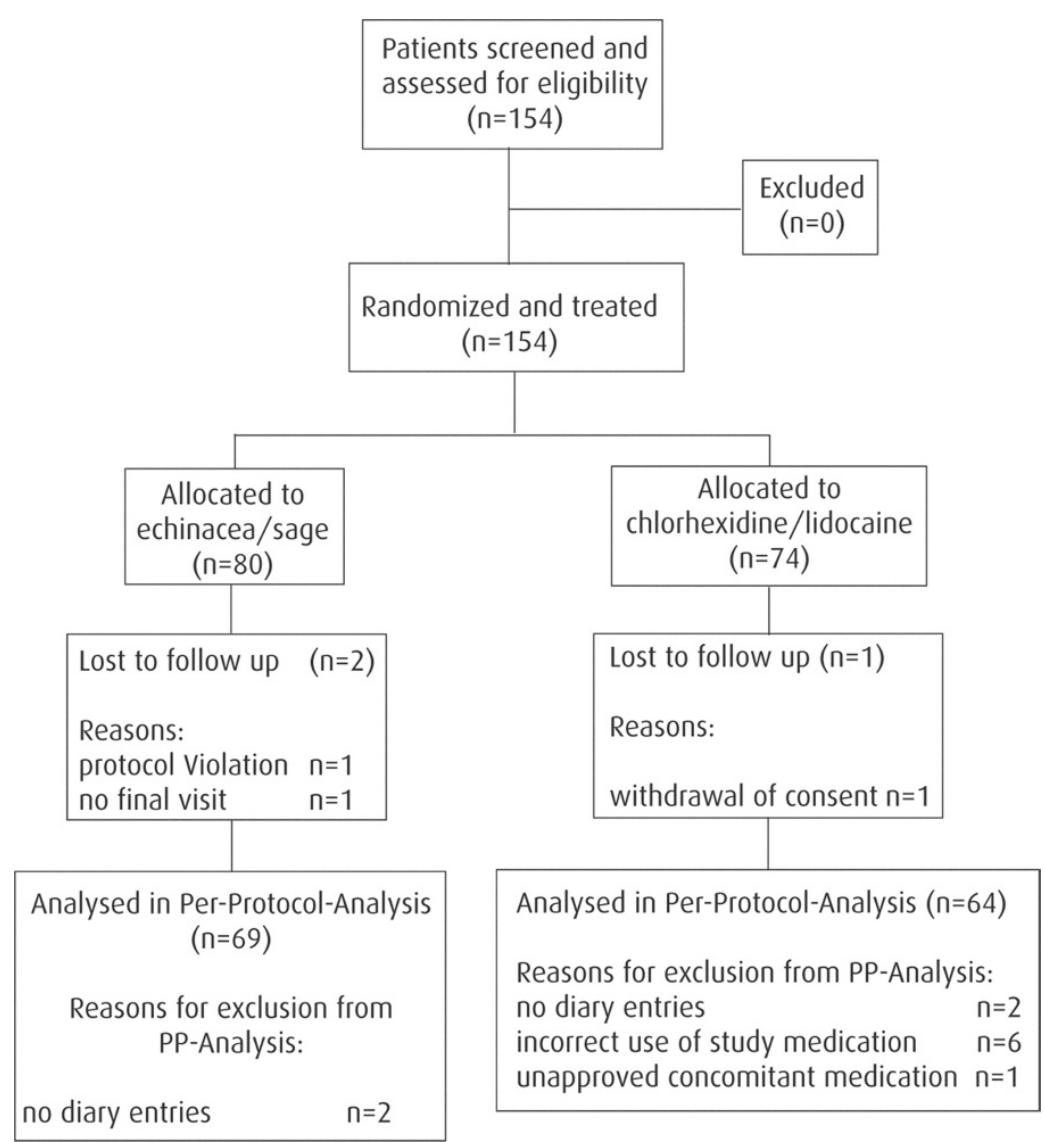

Fig. 1. Flow chart of participants in the trial. 
Table 1. Characteristics of patients in the two treatment groups for the per protocol population.

\begin{tabular}{|c|c|c|c|}
\hline & Echinacea/sage & Chlorhexidine/lidocaine & $\mathrm{P}$ value \\
\hline Number of patients & 69 & 64 & \\
\hline Mean age in years (SD) & $41.6(18.7)$ & $33.8(13.1)$ & $0.208^{1}$ \\
\hline Male & 23 & 20 & $>0.854^{2}$ \\
\hline Total throat score at baseline (SD) & $8.8(1.5)$ & $9.5(1.5)$ & 0.0041 \\
\hline Throat pain at baseline (100 mm VAS) (SD) & $72.6(17.9)$ & $77.1(19.9)$ & 0.0491 \\
\hline Mean study duration (SD) & 5.6 (2.1) days & 6.4 (4.8) days & 0.4941 \\
\hline Mean amount of verum used (SD) & $11.7(6.4) \mathrm{g}$ & $10.1(6.9) \mathrm{g}$ & \\
\hline Mean amount of placebo used (SD) & $7.8(5.4) \mathrm{g}$ & $14.2(10.5) \mathrm{g}$ & \\
\hline \multicolumn{4}{|l|}{ Blinding } \\
\hline Number of patients identifying verum correctly (\%) & $54(78.3)$ & $17(26.6)$ & \\
\hline Number of patients identifying placebo correctly (\%) & $52(75.4)$ & $15(23.4)$ & \\
\hline
\end{tabular}

${ }^{1}$ Wilcoxon two-sample tests

${ }^{2}$ Fisher's exact test

Table 2. Wilcoxon Test/Mann-Whitney statistic for day 1 to day 3 and combined hypothesis (per protocol collective).

\begin{tabular}{ccccc}
\hline Criterion & $\begin{array}{c}\text { Mann-Whitney } \\
\text { statistic }\end{array}$ & $\begin{array}{c}\text { Left-sided limit of } \\
\text { the confidence interval }\end{array}$ & $\begin{array}{c}\text { P value } \\
\text { (U-Test) }\end{array}$ & $\begin{array}{c}\mathrm{n} \\
\text { echinacea/sage } \\
\text { chlorhexidine/lidocaine }\end{array}$ \\
\hline Day 1 & 0.4949 & 0.4367 & 0.5681 & 64 \\
Day 2 & 0.5003 & 0.4174 & 0.4968 & 64 \\
Day 3 & 0.5298 & 0.4469 & 0.2408 & 64 \\
Global & 0.5083 & 0.4482 & 0.3928 & 69 \\
(Day 1- Day 3) & & & \\
\hline
\end{tabular}

\section{EFFICACY}

All efficacy data are shown for the per protocol population because it is needed for confirmatory analysis. Figure 2 shows the results for the primary parameter, the response rates in both treatment groups during the first 3 days. The echinacea/sage treatment was similar in efficacy to the chlorhexidine/lidocaine treatment for the 3 days together $(\mathrm{P}(\mathrm{x}<\mathrm{Y})=.5083)$ and for each single day of the first 3 days. The results for the Mann-Whitney-Statistics are given in Table 2.

The response rates for day 4 were $69.6 \%$ for echinacea/sage and $70.3 \%$ for chlorhexidine/lidocaine ( $P$ $=.5374)$; respectively for day 5 , they were $73.9 \%$ and $79.7 \%(\mathrm{P}=.7857)$.

Figure 3 illustrates the reduction in symptoms during the time course of the 5 days; there was no statistical difference between the two groups through the time period.

The time point at which $50 \%$ of patients in the echinacea/sage group were symptom free (total score $\leq 2$ ) was the evening of day 4 , while $50 \%$ of patients in the chlorhexidine/lidocaine group achieved symptom-free status the morning of day $5(\mathrm{P}=.6741)$.

At day 5, 50.7\% of the patients of the echinacea/sage and $56.3 \%$ of the chlorhexidine/lidocaine group were assessed to be symptom-free.

The pain recorded on a $100-\mathrm{mm}$ Visual Analogue Scale decreased under echinacea/sage from 72.6 to 7.3 $\mathrm{mm}$, and with chlorhexidine/lidocaine from 77.1 to $8.4 \mathrm{~mm}$ after 5 days of treatment $(\mathrm{P}=.6352)$.

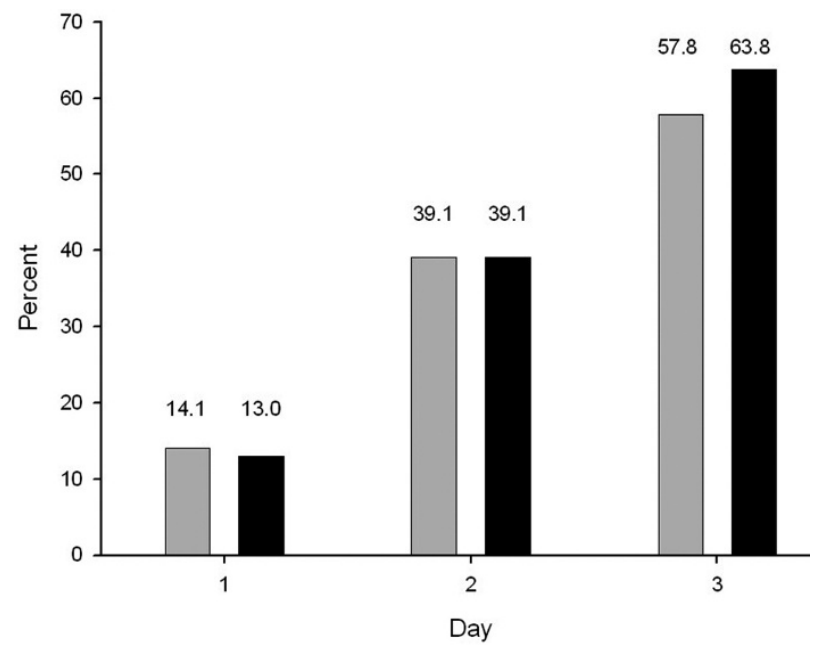

Fig. 2. Relative frequency of patients with reduction in the sore throat score by at least $50 \%$ during the first 3 days of treatment of the per protocol population $(n=133)$. The grey bars represent the chlorhexidine/lidocaine $(\mathrm{n}=64)$ and the black bars the echinacea/sage treatment $(n=69)$. $(P=.5083$, Mann-Whitney-Statistic).

In the echinacea/sage group, 25 patients took 6.2 ibuprofen tablets, and in the chlorhexidine/lidocaine group, 30 patients consumed 7.0 ibuprofen tablets during the observation period. A subanalysis with patients taking ibuprofen compared to those taking none showed no differences between the two populations in the efficacy parameters (data not shown). 


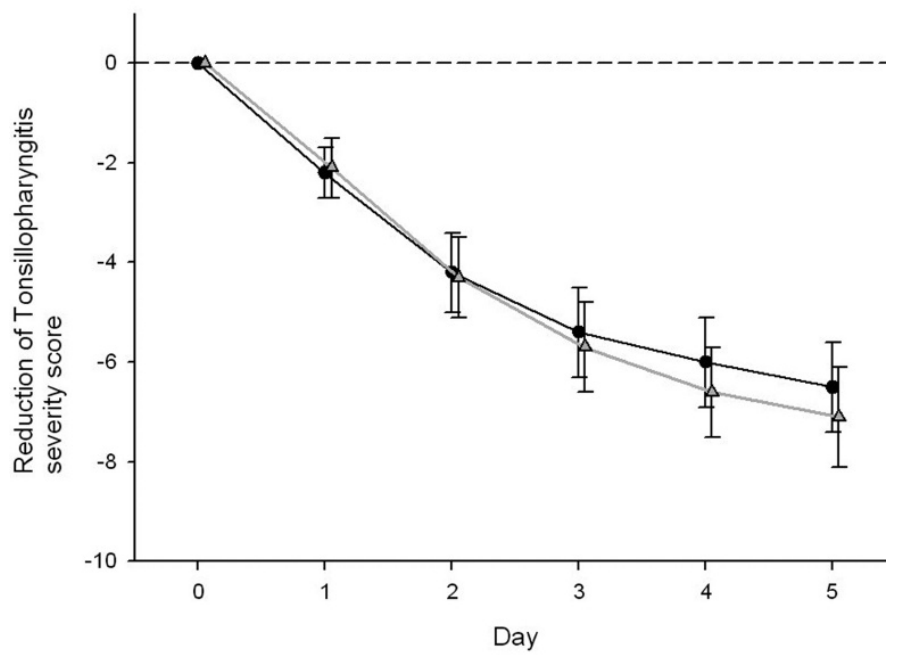

Fig. 3. Reduction of the total symptom score (per protocol collective) during 5 days of treatment of the per protocol population $(\mathrm{n}=133)$. The grey triangles show the chlorhexidine/lidocaine $(\mathrm{n}=64)$ and the black circles the echinacea/sage treatment $(n=69)$.
A

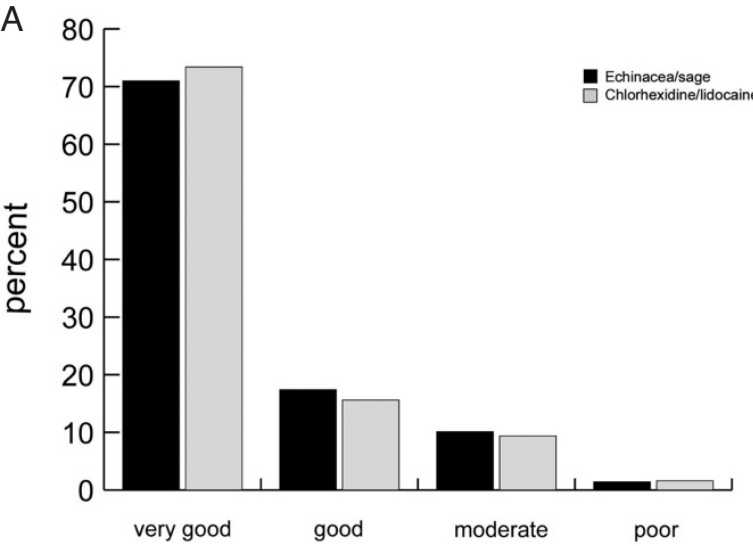

B

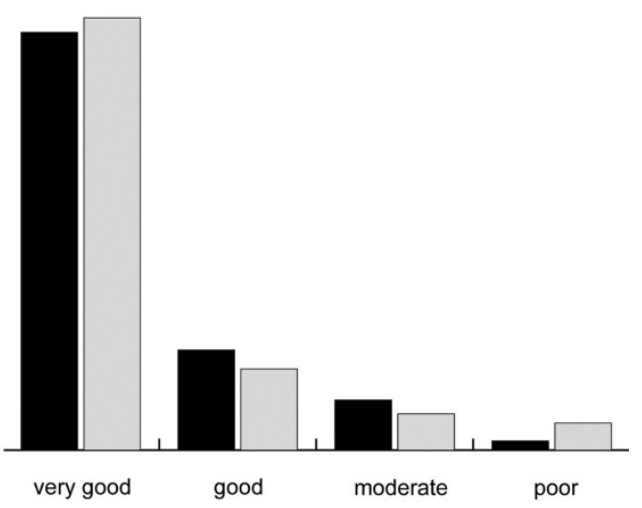

Fig. 4. Assessment of efficacy at the end of the treatment by investigators and patients (per protocol population, $\mathrm{n}=133$ ). $(\mathrm{A}=$ investigators, $\mathrm{B}=$ patients).

Investigators rated the efficacy as very good or good in $88.4 \%$ of patients using the echinacea/sage spray and in $89.1 \%$ of all patients using the chlorhexidine/lidocaine spray. Patients' efficacy ratings were similar, with "very good" or "good" in $89.9 \%$ of the echinacea/sage and $89.1 \%$ of the chlorhexidine/lidocaine cases (Fig. 4).

Because less spray was used in the chlorhexidine/lidocaine group, there was the possibility of an influence on the outcome in favor of the echinacea/sage spray; thus, we conducted a further analysis of the symptom reduction during the first 3 days with the amount of verum consumed in each group taken as the covariate. In this analysis, on days 1 and 2, a slight advantage of the echinacea/sage spray as in the primary analysis can be seen; on day 3, the advantage of chlorhexidine/lidocaine is more noticeable but not statistically significant $(\mathrm{P}=.610$; data not shown).

\section{SAFETY}

A total of six adverse events were observed among five patients. These were one rash on the mucosa, one burning sensation and dryness of the throat, and one patient with joint pains in the echinacea/sage group and one swelling of the tongue and a bitter taste in the mouth with chlorhexidine/lidocaine. All events except for the joint pains were seen to have a probable relationship to the study medication and were transient and of mild or moderate intensity, except for the bitter taste in the mouth reported by a patient in the chlorhexidine/lidocaine group, which was rated as severe.

The tolerability of the safety population was rated as good or very good for the chlorhexidine/lidocaine spray by $94.5 \%$ and for the echinacea/sage spray by $93.6 \%$ of the patients. The investigator ratings were almost the same with very good and good for $93.2 \%$ of all patients with chlorhexidine/lidocaine, and the same ratings for $96.2 \%$ of all patients in the echinacea/sage group. Vital parameters and laboratory values remained the same in both groups except for the C-reactive protein, which decreased under echinacea/sage treatment from 16.4 to $5.9 \mathrm{mg} / \mathrm{l}$ and under chlorhexidine/lidocaine from 23.0 to $6.4 \mathrm{mg} / 1$.

\section{Discussion}

Even though the occurrence of pharyngitis is very common and only $10-20 \%$ are streptococcal throat positive and require antibiotic treatment [4], only a few studies have been carried out on non-streptococcal sore throats, and no guidelines for studies or validated scores are available. We thus designed the trial based on the best available information. Nevertheless, we 
consider the symptom score used and the response criteria to be clinically relevant.

The choice of the reference treatment is always a matter of discussion. We chose the chlorhexidine/lidocaine spray in accordance with the relevant ICH guideline[13] because it has been on the market as a registered product in Switzerland since 1992 and is one of the leading products for treatment of acute sore throats in this country. Furthermore, the constituents chlorhexidine and lidocaine have long been in use and are well investigated. Chlorhexidine has been used over the past 25 years for a broad range of applications such as skin and surgical hand disinfection and for oral diseases and is used as gold standard among anti-plaque treatments. Its effectiveness is attributed to its bactericidal and bacteriostatic effects and its substantivity within the oral cavity.[6]

Lidocaine is a local anesthetic with a long history used topically for a variety of indications[14] .Even though chlorhexidine/lidocaine sprays are marketed in several countries, there are no studies available on a combination product in sore throats. Lidocaine has been investigated in studies with sore throat or in the oral mucosa. A study of $5 \%$ lidocaine ointment in healthy volunteers showed a significant pain reduction in the oral mucosa compared to placebo during $5 \mathrm{~min}-$ utes of induced pain.[15] A recent trial from Wonnemann[16] found that the use of $8 \mathrm{mg}$ lidocaine as tablets in patients with sore throat decreased the acute pain within the first 2 hours significantly compared to placebo $(\mathrm{P}<.001)$, a decrease that held for a longer treatment period of up to 48 hours after repeated intake of lidocaine tablets. The analgesic properties of lidocaine in the treatment of sore throat are thus clinically proven.

In our study, we showed that with the combination of sage and echinacea, the sore throat symptoms were reduced significantly. This finding is in line with clinical studies for sage and echinacea that examined the treatment of sore throats:

Hubbert et al. investigated the analgesic efficacy of three different sage spray formulations against placebo in patients with sore throats.[17] The pain during the first 2 hours was significantly lower than with placebo $(\mathrm{P}=.0021)$, and the pain measured over 3 days was significantly lower than placebo $(\mathrm{P}<.0001)$. The treatment was very well tolerated.

In four placebo-controlled clinical trials with an echinacea preparation, sore throat was also assessed as an efficacy parameter. All trials showed a benefit for echinacea but it was not always statistically significant[18-21]. These data indicate that with an echinacea preparation alone, no significant pain reduction in sore throats can be achieved, and as a consequence, a combination with sage is useful. But because reviews have confirmed the good prophylactic and acute efficacy of echinacea in upper respiratory tract infections,[11] echinacea also must contribute to the efficacy of the investigated throat spray.

The frequent use of ibuprofen can lead to the assumption that the study results are biased. This does not appear to be the case, however; in both treatment groups, about the same amount of ibuprofen was taken and, surprisingly, a subanalysis showed that the study results were the same whether patients took ibuprofen or not. Another interesting finding was that the majority of the patients in the chlorhexidine/lidocaine group thought they were actually receiving the echinacea/sage treatment. The amount of spray used shows that the patients also preferred the echinacea/sage treatment. We assume that this was mainly because of the taste of the echinacea/sage verum and placebo, which was preferred to the slightly sweetish taste of the chlorhexidine/lidocaine treatment. However, this finding also shows that the study was very well blinded, which is often hard to achieve with non-encapsulated study medications.

There is discussion in the treatment of pharyngitis of the extent to which antibiotics should be used. For practitioners and primary care institutions, there are simple and validated questionnaires available such as the McIsaac Score,[22] to differentiate between patients needing antibiotics and those needing simple analgesic treatment. Nevertheless, short-term use of analgesics also can be problematic. A study investigating ibuprofen, paracetamol, or aspirin use for up to 7 days in the treatment of cold symptoms and sore throats found an adverse event rate of $12 \%$ for ibuprofen, $15.7 \%$ for aspirin, and $12.3 \%$ for paracetamol.[23] In our study, we found for the echinacea/ sage treatment a low adverse event rate of $3.8 \%$. This is in line with other complementary medicinal products, which have proven to be well tolerated in the treatment of pharyngitis. [24] Because complementary medicines are generally well accepted and frequently used among patients[25], it is necessary to offer patients well-investigated and safe products.

\section{CONCLUSIONS}

The echinacea/sage spray demonstrated equivalent efficacy compared to the chlorhexidine/lidocaine treatment and was very well tolerated. It is a recommendable alternative to common NSAIDs or other synthetic products in the treatment of acute sore throats.

Competing interests: Andy Suter is head of the medical department of A. Vogel Bioforce AG, Roggwil, Switzerland which financed this clinical trial. He was involved in planning and supervision of the carrying out of the clinical trial. All other authors have no competing interests.

Author's contributions: $\mathrm{DB}$ and ASu set up the study design, ASu supervised the clinical trial carried out by an independent contract research organisation, appletree ag, Winterthur, Switzerland. PK provided the randomisation list, the procedure for the statistical analysis and the estimated number of patients and performed the statistical analysis. ASc reviewed the final data and commented the study report. All authors read and approved the final manuscript.

Acknowledgments: We would like to thank the following investigators for participating in the trial : Doris Geiger, Au; Dominique Kähler, Wil; Heidi Martin, Gossau; Huldrych Steinemann, St. Gallen; Martin Schütz, Zürich; Ferdinand Jaggi, Zürich; Micha Steigbügel, Wald; Christoph Zeller, Rüti; Markus Wieser, Winterthur; Roland Meister, Winterthur; Markus Burla, Zürich.

Blerim Krasniqi from the product development of A. Vogel Bioforce AG for the preparation of the study medication and 
the placebos and appletree AG, Winterthur, Switzerland, for the proper carrying out of the trial.

This study has been sponsored by

A. Vogel Bioforce AG, Roggwil, Switzerland.

Trial Registry Number: http://clinicaltrials.gov; NCT00707902

\section{REFERENCES}

1 Wei JL, Kasperbauer JL, Weaver AL, Boggust AJ: Efficacy of single-dose dexamethasone as adjuvant therapy for acute pharyngitis. Laryngoscope. 2002 Jan; 112(1):87-93.

2 van Driel ML, De Sutter A, Deveugele M, Peersman W, Butler CC, De Meyere M, De Maeseneer J, Christiaens T: Are sore throat patients who hope for antibiotics actually asking for pain relief? Ann Fam Med. 2006 Nov-Dec; 4(6):494-9.

3 Vincent MT, Celestin N, Hussain AN: Pharyngitis. Am Fam Physician. 2004 Mar 15; 69(6):1465-70. Review.

4 Bertin L, Pons G, d'Athis P et al: Randomized, doubleblind, multicenter, controlled trial of ibuprofen versus acetaminophen (paracetamol) and placebo for treatment of symptoms of tonsillitis and pharyngitis in children.J Pediatr. 1991 Nov; 119(5):811-4.

5 Eccles R, Loose I, Jawad M, Nyman L: Effects of acetylsalicylic acid on sore throat pain and other pain symptoms associated with acute upper respiratory tract infection. Pain Med. 2003 Jun; 4(2):118-24.

6 Moshrefi A: Chlorhexidine. J West Soc Periodontol Periodontal Abstr. 2002, 50(1):5-9.

7 Shah SA, Sander S, White CM, Rinaldi M, Coleman CI: Evaluation of echinacea for the prevention and treatment of the common cold: a meta-analysis. Lancet Infect Dis. 2007 Jul ; 7(7):473-80. Review.

8 Gertsch J, Schoop R, Kuenzle U, Suter A : Echinacea alkylamides modulate TNF-alpha gene expression via cannabinoid receptor $\mathrm{CB} 2$ and multiple signal transduction pathways. FEBS Lett. 2004 Nov 19 ; 577(3):563-9

9 Hudson J, Altamirano M: The application of DNA micro-arrays (gene arrays) to the study of herbal medicines. J Ethnopharmacol. 2006 Nov 3 ; 108(1):2-15.

10 ESCOP: Sage leaf, Salviae officinalis folium. ESCOP monographs. 2nd edition. Exeter, Stuttgart, New York: ESCOP, Georg Thieme Verlag, Thieme New York, 2003: 339-344

11 Bereznoy VV, Riley DS, Wassmer G, Heger M: Efficacy of extract of Pelargonium sidoides in children with acute non-group A beta-hemolytic streptococcus tonsillopharyngitis: a randomized, double-blind, placebo-controlled trial. Altern Ther Health Med. 2003; 9(5):68-79

12 Wei, L.J. and Lachin, J.M: Two-sample asymptotically distribution-free tests for incomplete multivariate obeservations. Journal of the American Statistical Association. 1984; 79 (387): $653-661$

13 European Medicines Agency EMEA: CPMP/ICH/ 363/96. ICH Topic E 9 Statistical Principles for Clinical Trials. Note for guidance on statistical principles for clinical trials (CPMP/ICH/363/96). September 1998

14 MacKenzie TA, Young ER: Local anesthetic update. Anesth Prog. 1993; 40(2):29-34.

15 Holst A, Evers H: Experimental studies of new topical anaesthetics on the oral mucosa. Swed Dent J. 1985; 9(5): 185-91.

16 Wonnemann M, Helm I, Stauss-Grabo M et al: Lidocaine $8 \mathrm{mg}$ sore throat lozenges in the treatment of acute pharyngitis. A new therapeutic option investigated in comparison to placebo treatment. Arzneimittelforschung. 2007; 57(11):689-97.
17 Hubbert M, Sievers H, Lehnfeld R, Kehrl W: Efficacy and tolerability of a spray with Salvia officinalis in the treatment of acute pharyngitis - a randomised, doubleblind, placebo-controlled study with adaptive design and interim analysis. Eur J Med Res. 2006 Jan 31; 11(1):20-6

18 Schulten B, Bulitta M, Ballering-Brühl B, Köster U, Schäfer M: Efficacy of Echinacea purpurea in patients with a common cold. A placebo-controlled, randomised, double-blind clinical trial. Arzneimittelforschung. 2001; 51(7):563-8

19 Goel V, Lovlin R, Barton R, Lyon MR, Bauer R, Lee TD, Basu TK: Efficacy of a standardized echinacea preparation (Echinilin) for the treatment of the common cold: a randomized, double-blind, placebo-controlled trial. J Clin Pharm Ther. 2004 Feb; 29(1):75-83.

20 Sperber SJ, Shah LP, Gilbert RD, Ritchey TW, Monto AS: Echinacea purpurea for prevention of experimental rhinovirus colds. Clin Infect Dis. 2004 May 15; 38(10): 1367-71

21 Braunig B: Echinacea purpurea radix for strengthening the immune response in flu-like infections. Z Phytother 1992; 13:7-13

22 McIsaac WJ, White D, Tannenbaum D, Low DE: A clinical score to reduce unnecessary antibiotic use in patients with sore throat. CMAJ. 1998 Jan 13; 158(1):75-83.

23 Moore N, Le Parc JM, van Ganse E, Wall R, Schneid H, Cairns R: Tolerability of ibuprofen, aspirin and paracetamol for the treatment of cold and flu symptoms and sore throat pain. Int J Clin Pract. 2002 Dec; 56(10):732-4

24 Hamre HJ, Fischer M, Heger M et al: Anthroposophic vs. conventional therapy of acute respiratory and ear infections: a prospective outcomes study. Wien Klin Wochenschr. 2005 Apr; 117(7-8):256-68.

25 Barnes P, Powell-Griner E, McFann K, Nahin R: Complementary and alternative medicine use among adults: United States. CDC Advance Data Report \#343. 2004

Received: December 8, 2008 / Accepted: July 21, 2009

Address for correspondence:

PD Dr. med. Dr. h. c. Andreas Schapowal

Consultant in ear, nose, and throat medicine

Allergy Clinic

CH-7302 Landquart

Switzerland

Tel.: $\quad+41-81-3224040$

Fax: +41-81-3223262

E-mail: andreas@schapowal.ch

Daniel Berger

Dr. pharm.

CH-3054 Schüpfen

Switzerland

Peter Klein

Dipl. mathematician

Statistician

d.s.h. statistical services $\mathrm{GmbH}$

85296 Rohrbach

Germany

Andy Suter

Dipl. biologist

Head of Medical Department

A. Vogel Bioforce AG

CH-9325 Roggwil

Switzerland

Tel.: +41 4546 203; Fax: + 414546164

E-mail: a.suter@bioforce.ch 